\title{
Straight-line Instruction Sequence Completeness for Total Calculation on Cancellation Meadows
}

\author{
Jan A. Bergstra • Inge Bethke
}

Published online: 27 May 2010

(C) The Author(s) 2010. This article is published with open access at Springerlink.com

\begin{abstract}
A combination of program algebra with the theory of meadows is designed leading to a theory of computation in algebraic structures. It is proven that total functions on cancellation meadows can be computed by straight-line programs using at most five auxiliary variables. A similar result is obtained for signed meadows.
\end{abstract}

Keywords Program algebra $\cdot$ Instruction sequences $\cdot$ Execution of programs · Straight-line programs · Division-by-zero $\cdot$ Fields $\cdot$ Meadows $\cdot$ Equational specification $\cdot$ Calculation in meadows

\section{Introduction}

Program algebra is an approach to the formal description of the semantics of programming languages. It is a framework that permits algebraic reasoning about programs and has been investigated in various settings (see e.g. [4, 13-15, 19]).

The theory of fields is a very active area which is not only of great theoretical interest but has also found applications both within mathematics-combinatorics and algorithm analysis - as well as in engineering sciences and, in particular, in coding theory and sequence design. Unfortunately, since fields are not axiomatized by equations only, Birkhoff's Theorem fails, i.e. fields do not constitute a variety: they are not closed under products, subalgebras, and homomorphic images. In [10], the concept of meadows was introduced, structures very similar to fields-the considerable difference being that meadows enjoy a total multiplicative inversion and do form a variety.

J.A. Bergstra $\cdot$ I. Bethke (凶)

Section Theory of Computer Science, Informatics Institute, University of Amsterdam, Amsterdam, The Netherlands

e-mail: I.Bethke@uva.nl

url: http://www.science.uva.nl inge

J.A. Bergstra

url: http://www.science.uva.nl janb 
The aim of this paper is to combine these two areas of research in order to create a theory of computation in algebraic structures which can be used to investigate questions of definability and complexity.

Many computations in applied mathematics can be formulated as computations on fields. In many cases such computations terminate on all inputs yielding total functions. Replacing fields by meadows, which simplify their equational logic, we investigate properties of instruction sequences which compute total functions on meadows.

Cancellation meadows-meadows which in addition satisfy the inverse law known from the theory of fields - constitute a very general datatype for computation. One may easily simulate Booleans in a cancellation meadow, and numbers (perhaps modulo a squarefree number) of rationals with arbitrary precision are also available. For instance algorithms meant for digital signal processing can be modeled within a cancellation meadow. Although we will not provide a series of examples here, we consider the generality of cancellation meadows as a datatype for practical computation beyond reasonable doubt. Computations over cancellation meadows can be imagined to take place on a large scale in embedded devices of various kinds. Executable programs for such devices need to satisfy constraints which can hardly be formulated in the high level notation from which such programs are obtained by means of compilation. These considerations justify the analysis of algorithms over cancellation meadows and in particular their representation in terms of low level program notations. The objective of our paper is to determine a basis for such investigations by proving the remarkable expressive power of straight-line programs. These kind of programs have been amply investigated and simplification and equivalence problems for several classes of straight-line programs over varying instruction sets are known (see e.g. $[16,17])$.

We will prove a standard form result for algorithms that terminate on each cancellation meadow. As a general class we consider algorithms that can be represented by means of instruction sequences with tests and jumps. That class of programs provides a reasonable abstraction of assembly level programs. All such algorithms can be transformed into an equivalent straight-line program making use of a bounded set of program variables for meadow quantities. Thus jumps, tests and loops and additional program variables do not add to the expressive power of the program notation in this case.

The paper is organized as follows. In the next section we recall the basics of program algebra, thread algebra and meadows. Here the notion of program algebra refers to the concept introduced in [4] which focuses on instruction sequences. In Sect. 3 we introduce instruction sequences for functions on the rational numbers. The main theorem is proven in Sect. 4. We prove that total functions on cancellation meadows can be represented by a normal form without tests and jumps which uses at most five auxiliary variables. This result is extended to signed cancellation meadows - cancellation meadows that presuppose an ordering of its domain-in Sect. 5.

\section{The Basics of Program Algebra, Thread Algebra and Meadows}

In this section we recall program algebra, thread algebra and meadows. 


\subsection{Program Algebra}

The program algebra PGA was introduced in [4].

Assume $A$ is a set of constants with typical elements $\mathrm{a}, \mathrm{b}, \mathrm{c}, \ldots$. Instruction sequences are of the following form $(\mathrm{a} \in A, k \in \mathbb{N})$ :

$$
I::=\mathrm{a}|+\mathrm{a}|-\mathrm{a}|\# k| !|I ; I| I^{\omega} .
$$

The first five forms above are called primitive instructions. These are

- basic instructions a which prescribe behaviours that are considered indivisible and executable in finite time, and which return upon execution a Boolean reply value,

- test instructions obtained from basic instructions by prefixing them with either a + (positive test instruction) or a - (negative test instruction) which control subsequent execution via the reply of their execution by continuing execution either with the next instruction or with the instruction thereafter,

- jump instructions \#k which prescribe to jump $k$ instructions ahead-if $k=0$, this jump is (zero steps forward) to the instruction itself, i.e. inaction will result-and generate no observable behaviour, and

- the termination instruction! which prescribes successful termination, an event that is taken to be observable.

Instruction sequences are obtained from primitive instructions using two types of composition mechanisms, namely

- concatenation: if $I$ and $J$ are instruction sequences, then so is

$$
I ; J
$$

which informally is the instruction sequence that lists $J$ 's instructions right after those of $I$, and

- repetition: if $I$ is an instruction sequence, then

$$
I^{\omega}
$$

is the instruction sequence that informally repeats $I$ forever, thus $I ; I ; I ; \ldots$.

Finite instruction sequences are instruction sequences without repetition; straightline instruction sequences are finite instruction sequences without tests and jumps.

In PGA, different types of equality are discerned, the most simple of which is single-pass congruence, identifying sequences that execute identical instructions. For finite instruction sequences, single-pass congruence boils down to the associativity of concatenation, and is axiomatized by

$$
(X ; Y) ; Z=X ;(Y ; Z) .
$$

In the sequel we leave out brackets in repeated concatenations. In the case of infinite instruction sequences, additional axioms are needed. Define $X^{1}=X$ and for $n>0$, $X^{n+1}=X ; X^{n}$. According to [4], single-pass congruence for arbitrary instruction sequences is axiomatized by the axiom schemes PGA1-PGA4 in Table 1. 
Table 1 PGA-axioms for structural congruence $(n, m, k \geq 0)$

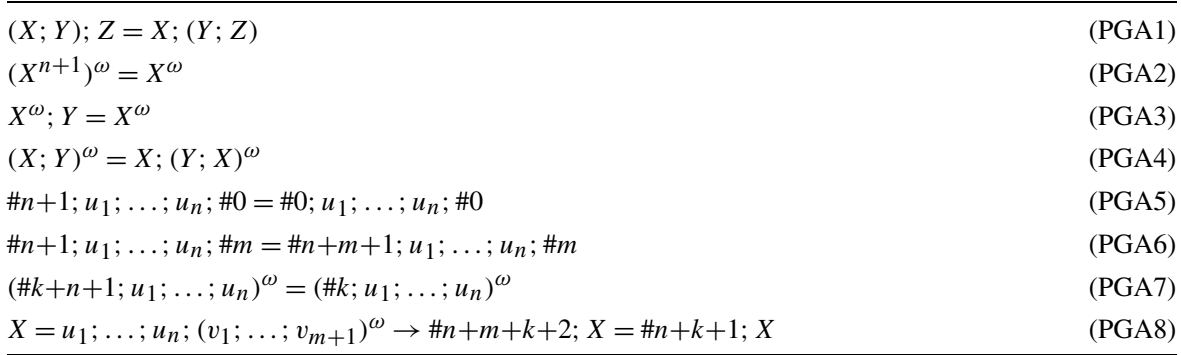

Using the axioms PGA1-PGA4 and thus preserving single-pass congruence, each instruction sequence can be rewritten into an instruction sequence of the form

- $I$ with $I$ finite, or

- $I ; J^{\omega}$ with $I$ and $J$ finite.

Instruction sequences of the two forms above are said to be in first canonical form.

Instruction sequences in first canonical form can be converted into second canonical form: a first canonical form in which no chained jumps occur, i.e., jumps to jump instructions cannot happen (apart from \#0 which is a jump of length 0 to the instruction itself), and in which each non-chaining jump into the repeating part is minimized. The associated congruence $=_{s c}$ is called structural congruence and is axiomatized in Table 1. Note that axiom PGA8 is an equational axiom, the implication is only used to enhance readability. Two examples, of which the right-hand sides are in second canonical form:

$$
\begin{gathered}
\# 2 ; \mathrm{a} ;(\# 5 ; \mathrm{b} ;+\mathrm{c})^{\omega}={ }_{s c} \# 4 ; \mathrm{a} ;(\# 2 ; \mathrm{b} ;+\mathrm{c})^{\omega}, \\
+\mathrm{a} ; \# 2 ;(+\mathrm{b} ; \# 2 ;-\mathrm{c} ; \# 2)^{\omega}={ }_{s c}+\mathrm{a} ; \# 0 ;(+\mathrm{b} ; \# 0 ;-\mathrm{c} ; \# 0)^{\omega} .
\end{gathered}
$$

For each instruction sequence there exists a structurally equivalent second canonical form. For more information on PGA we refer to [4, 18].

\subsection{Thread Algebra}

Thread algebra is the behavioural semantics for PGA and was introduced in e.g. [1, 4] under the name Polarized Process Algebra.

Finite threads are defined inductively by:

S stop, the termination thread,

D inaction or deadlock, the inactive thread,

$T \unlhd \mathrm{a} \unrhd T^{\prime} \quad$ the postconditional composition of $T$ and $T^{\prime}$ for action a, where $T$ and $T^{\prime}$ are finite threads and $\mathrm{a} \in A$.

The behaviour of the thread $T \unlhd \mathrm{a} \unrhd T^{\prime}$ starts with the action a and continues as $T$ upon reply true to a, and as $T^{\prime}$ upon reply false. Note that finite threads always 
end in $\mathrm{S}$ or $\mathrm{D}$. We use action prefix a $\circ T$ as an abbreviation for $T \unlhd \mathrm{a} \unrhd T$ and take o to bind strongest.

For every finite thread there exists a finite upper bound to the number of consecutive actions it can perform. The approximation operator $\pi_{n}$ gives the behaviour up to depth $n$ and is defined by

1. $\pi_{0}(T)=\mathrm{D}$,

2. $\pi_{n+1}(\mathrm{~S})=\mathrm{S}$,

3. $\pi_{n+1}(\mathrm{D})=\mathrm{D}$, and

4. $\pi_{n+1}\left(T \unlhd a \unrhd T^{\prime}\right)=\pi_{n}(T) \unlhd a \unrhd \pi_{n}\left(T^{\prime}\right)$

for finite threads $T, T^{\prime}$ and $n \in \mathbb{N}$. Infinite threads are obtained as projective sequences of finite threads of the form $\left(T_{n}\right)_{n \in \mathbb{N}}$, where for every $n \in \mathbb{N}, \pi_{n}\left(T_{n+1}\right)=T_{n}$.

Upon its execution, a basic or test instruction yields the equally named action in a postconditional composition. Thread extraction on PGA, notation

with $X$ an instruction sequence in second canonical form, is defined by the thirteen equations in Table 2. In particular, note that upon the execution of a positive test instruction $+\mathrm{a}$, the reply true to a prescribes to continue with the next instruction and false to skip the next instruction and to continue with the instruction thereafter; if no such instruction is available, deadlock occurs. For the execution of a negative test instruction $-\mathrm{a}$, subsequent execution is prescribed by the complementary replies.

For an instruction sequence in second canonical form, these equations either yield a finite thread, or a so-called regular thread, i.e., a finite state thread in which infinite paths can occur. Each regular thread can be specified (defined) by a finite number of recursive equations. As an example, the regular thread $T$ specified by

$$
\begin{aligned}
T & =\mathrm{a} \circ T^{\prime}, \\
T^{\prime} & =\mathrm{c} \circ T^{\prime} \unlhd \mathrm{b} \unrhd(\mathrm{S} \unlhd \mathrm{d} \unrhd T)
\end{aligned}
$$

Table 2 Equations for thread extraction, where a ranges over the basic instructions, and $u$ over the primitive instructions $(k \in \mathbb{N})$

\begin{tabular}{ll}
\hline$|!|=\mathrm{S}$ & $|! ; X|=\mathrm{S}$ \\
$|\mathrm{a}|=\mathrm{a} \circ \mathrm{D}$ & $|\mathrm{a} ; X|=\mathrm{a} \circ|X|$ \\
$|+\mathrm{a}|=\mathrm{a} \circ \mathrm{D}$ & $|+\mathrm{a} ; X|=|X| \unlhd \mathrm{a} \unrhd|\# 2 ; X|$ \\
$|-\mathrm{a}|=\mathrm{a} \circ \mathrm{D}$ & $|-\mathrm{a} ; X|=|\# 2 ; X| \unlhd \mathrm{a} \unrhd|X|$ \\
& $|\# 0 ; X|=\mathrm{D}$ \\
$|\# k|=\mathrm{D}$ & $|\# 1 ; X|=|X|$ \\
& $|\# k+2 ; u|=\mathrm{D}$ \\
& $|\# k+2 ; u ; X|=|\# k+1 ; X|$ \\
\hline
\end{tabular}


can be defined by $\mid \mathrm{a} ;(+\mathrm{b} ; \# 2 ; \# 3 ; \mathrm{c} ; \# 4 ;+\mathrm{d} ; \text { !; a })^{\omega} \mid$. A picture of this thread is

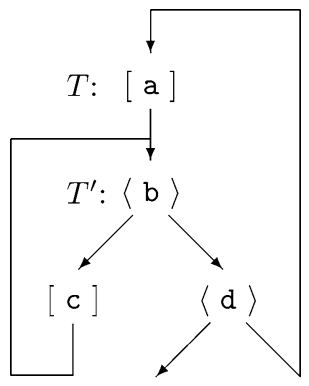

S

This thread can also given be by the projective sequence $\left(\pi_{n}(T)\right)_{n \in \mathbb{N}}$ where

$$
\begin{aligned}
& \pi_{0}(T)=\mathrm{D}, \\
& \pi_{1}(T)=\mathrm{a} \circ \mathrm{D}, \\
& \pi_{2}(T)=\mathrm{a} \circ \mathrm{b} \circ \mathrm{D}, \\
& \pi_{3}(T)=\mathrm{a} \circ(\mathrm{c} \circ \mathrm{D} \unlhd \mathrm{b} \unrhd \mathrm{d} \circ \mathrm{D})
\end{aligned}
$$

and $\pi_{n+4}(T)=\mathrm{a} \circ\left(\mathrm{c} \circ \pi_{n+1}\left(T^{\prime}\right) \unlhd \mathrm{b} \unrhd\left(\mathrm{S} \unlhd \mathrm{d} \unrhd \pi_{n+1}(T)\right)\right)$. Observe that thread extraction of straight-line instruction sequences yields finite and test-free threads.

For basic information on thread algebra we refer to $[2,18]$; more advanced matters, such as an operational semantics for thread algebra, are discussed in [5]. We here only mention the fact that each regular thread can be specified in PGA, and, conversely, that each PGA-program defines a regular thread.

\subsection{Meadows}

A meadow $[3,10]$ is a commutative ring with unit equipped with a total unary operation (_) $)^{-1}$ named restricted inverse that satisfies the two equations

$$
\begin{gathered}
\left(x^{-1}\right)^{-1}=x, \\
x \cdot\left(x \cdot x^{-1}\right)=x \quad(\text { RIL }) .
\end{gathered}
$$

Here RIL abbreviates Restricted Inverse Law. We write $M d$ for the set of axioms in Table 3 .

In the meadow $\mathbb{Q}$ of rational numbers, every element has a restricted inverse. If $x \neq 0$, the inverse is just the "regular" inverse, and $0^{-1}=0$. Another example is ring $\mathbb{Z} / 6 \mathbb{Z}$ with elements $\{0,1,2, \ldots, 5\}$ where arithmetic is performed modulo 6 . We find that every element has a restricted inverse as follows:
$(0)^{-1}=0$,
$(1)^{-1}=1$,
$(2)^{-1}=2$,
$(3)^{-1}=3$,
$(4)^{-1}=4$
$(5)^{-1}=5$. 
Table 3 The set $M d$ of axioms for meadows

$$
\begin{aligned}
& (x+y)+z=x+(y+z) \\
& x+y=y+x \\
& x+0=x \\
& x+(-x)=0 \\
& (x \cdot y) \cdot z=x \cdot(y \cdot z) \\
& x \cdot y=y \cdot x \\
& 1 \cdot x=x \\
& x \cdot(y+z)=x \cdot y+x \cdot z \\
& \left(x^{-1}\right)^{-1}=x \\
& x \cdot\left(x \cdot x^{-1}\right)=x
\end{aligned}
$$

A characterization of finite meadows can be found in [12]. From the axioms in $M d$ the following identities are derivable:

$$
\begin{aligned}
(0)^{-1} & =0 \\
(-x)^{-1} & =-\left(x^{-1}\right), \\
(x \cdot y)^{-1} & =x^{-1} \cdot y^{-1}, \\
0 \cdot x & =0 \\
x \cdot-y & =-(x \cdot y), \\
-(-x) & =x
\end{aligned}
$$

We write $\Sigma_{m}=\left(0,1,+, \cdot,-,{ }^{-1}\right)$ for the signature of meadows and $\operatorname{Ter}\left(\Sigma_{m}, X\right)$ for the set of open meadow terms with free variables in $X$. For $t, u \in \operatorname{Ter}\left(\Sigma_{m}, X\right)$ we shall often write $1 / t$ or

$$
\frac{1}{t}
$$

for $t^{-1}, t u$ for $t \cdot u, t / u$ for $t \cdot 1 / u, t-u$ for $t+(-u)$, and freely use numerals $n$-abbreviating $\underbrace{1+\cdots+1}_{n}$-and exponentiation with integer exponents as in $t^{n}$. We shall further write

$$
1_{x} \text { for } \frac{x}{x} \text { and } 0_{x} \text { for } 1-1_{x}
$$

so, $0_{0}=1_{1}=1,0_{1}=1_{0}=0$, and for all terms $t$,

$$
0_{t}+1_{t}=1
$$

We write $\Sigma_{r}=(0,1,+, \cdot,-)$ for the signature of rings. A polynomial is an expression over $\Sigma_{r}$, thus without inverse operator. Note that every polynomial can be represented as a finite sum of monomials, i.e. products of variables with integer coefficients. Meadow terms enjoy a particular standard representation which was introduced in [8]. 
Definition 2.1 A term $t \in \operatorname{Ter}\left(\Sigma_{m}, X\right)$ is a Standard Meadow Form (SMF) if, for some $n \in \mathbb{N}, t$ is an SMF of level $n$. SMFs of level $n$ are defined as follows:

1. SMF of level 0: each expression of the form $s / t$ with $s$ and $t$ ranging over polynomials,

2. SMF of level $n+1$ : each expression of the form

$$
0_{t^{\prime}} \cdot s+1_{t^{\prime}} \cdot t
$$

with $t^{\prime}$ ranging over polynomials and $s$ and $t$ over SMFs of level $n$.

Each meadow term is provably equal to a Standard Meadow Form in the same variables.

Theorem 2.2 For each $t \in \operatorname{Ter}\left(\Sigma_{m}, X\right)$ there exist an SMF $t_{\mathrm{SMF}}$ in the same variables such that $M d \vdash t=t_{\mathrm{SMF}}$.

Proof See [8].

It follows that every meadow term is provably equal to a sum of quotients of polynomials.

Corollary 2.3 For every $t \in \operatorname{Ter}\left(\Sigma_{m}, X\right)$ there exist polynomials $s_{0}, t_{0}, \ldots, s_{n}, t_{n}$ such that

$$
M d \vdash t=\frac{s_{0}}{t_{0}}+\cdots+\frac{s_{n}}{t_{n}} .
$$

Proof Let $t_{\mathrm{SMF}}$ be a SMF of $t$. We employ induction on its level $n$. If $n=0$ then $t_{\mathrm{SMF}}=s_{0} / t_{0}$ with $s_{0}$ and $t_{0}$ polynomials. Assume $n=m+1$. Then $t_{\mathrm{SMF}}=$ $0_{t^{\prime \prime}} \cdot s+1_{t^{\prime \prime}} \cdot t^{\prime}$ where $t^{\prime \prime}$ is a polynomial, and $s, t^{\prime}$ are SMF's of level $m$. By the induction hypothesis $s=s_{0} / t_{0}+\cdots+s_{k} / t_{k}$ and $t^{\prime}=u_{0} / v_{0}+\cdots+u_{l} / v_{l}$ with $s_{0}, t_{0}, \ldots, s_{k}, t_{k}, u_{1}, v_{1}, \ldots, u_{l}, v_{l}$ polynomials. Then

$$
\begin{aligned}
t_{\mathrm{SMF}} & =0_{t^{\prime \prime}} \cdot s+1_{t^{\prime \prime}} \cdot t^{\prime} \\
& =\left(1-\frac{t^{\prime \prime}}{t^{\prime \prime}}\right) \cdot s+\frac{t^{\prime \prime}}{t^{\prime \prime}} \cdot t^{\prime} \\
& =s-\left(\frac{t^{\prime \prime} s_{0}}{t^{\prime \prime} t_{0}}+\cdots+\frac{t^{\prime \prime} s_{k}}{t^{\prime \prime} t_{k}}\right)+\frac{t^{\prime \prime} u_{0}}{t^{\prime \prime} v_{0}}+\cdots+\frac{t^{\prime \prime} u_{l}}{t^{\prime \prime} v_{l}} \\
& =s+\frac{-t^{\prime \prime} s_{0}}{t^{\prime \prime} t_{0}}+\cdots+\frac{-t^{\prime \prime} s_{k}}{t^{\prime \prime} t_{k}}+\frac{t^{\prime \prime} u_{0}}{t^{\prime \prime} v_{0}}+\cdots+\frac{t^{\prime \prime} u_{l}}{t^{\prime \prime} v_{l}}
\end{aligned}
$$

and the last term is again a sum of quotients of polynomials.

The term cancellation meadow was introduced in [9] for a zero-totalized fielda field in which $0^{-1}=0$. Cancellation meadows satisfy in addition the so-called cancellation axiom

$$
x \neq 0 \& x \cdot y=x \cdot z \longrightarrow y=z
$$


An equivalent version of the cancellation axiom is the Inverse Law (IL), i.e., the conditional axiom

$$
x \neq 0 \longrightarrow x \cdot x^{-1}=1 \quad(I L) .
$$

So $I L$ states that there are no proper zero divisors. (Another equivalent formulation of the cancellation property is $x \cdot y=0 \longrightarrow x=0$ or $y=0$.) The rationals $\mathbb{Q}$ form a cancellation meadow, $\mathbb{Z} / 6 \mathbb{Z}$ does not.

\section{Calculation on Cancellation Meadows}

Instruction sequences for functions on the rational numbers are designed in such a way that computations can be performed only with the aid of auxiliary variables to which initially the input values are copied, and from which the final values are copied to the output.

\section{Definition 3.1}

1. We distinguish two infinite, countable sets of input and auxiliary variables $\operatorname{Var}_{\text {in }}=$ $\left\{x_{i} \mid i \in \mathbb{N}\right\}$ and $\operatorname{Var}_{a u x}=\left\{a_{i} \mid i \in \mathbb{N}\right\}$, and a single output variable $y$. Var denotes the union of these variables.

2. The instruction set $\operatorname{Ins}(\mathbb{Q})$ - instructions on the rational numbers - consists of the following input, auxiliary and output instructions:

$$
\begin{aligned}
\operatorname{Ins}(\mathbb{Q})_{\text {in }}= & \left\{a . \operatorname{cp}(x) \mid a \in \operatorname{Var}_{\text {aux }} \& x \in \operatorname{Var}_{\text {in }}\right\}, \\
\operatorname{Ins}(\mathbb{Q})_{\text {aux }}= & \left\{a . \text { set }: 0, \text { a.set }: 1, a . \text { set }: \text { ai, a.set }: \operatorname{mi} \mid a \in \operatorname{Var}_{a u x}\right\} \\
& \cup\left\{a . \text { set }: \mathrm{a}\left(a^{\prime}\right), \text { a.set }: \mathrm{m}\left(a^{\prime}\right), \mid a, a^{\prime} \in \operatorname{Var}_{\text {aux }}\right\} \\
& \cup\left\{\text { a.test }: 0 \mid a \in \operatorname{Var}_{a u x}\right\}, \\
\operatorname{Ins}(\mathbb{Q})_{\text {out }}= & \left\{y \cdot \operatorname{cp}(a) \mid a \in \operatorname{Var}_{a u x}\right\} .
\end{aligned}
$$

Here $\mathrm{a} i$ and $\mathrm{mi}$ refer to the unary meadow operations of additive and multiplicative inversion, and $a$ and $m$ to binary addition and multiplication. The intended meaning of these instructions is depicted in Table 4: $a \cdot \operatorname{cp}(x)$ copies the value of the input

Table 4 The instruction set and its informal semantics

\begin{tabular}{ll}
\hline a.cp $(x)$ & {$[a \Leftarrow x]$} \\
a.set: 0 & {$[a \Leftarrow 0]$} \\
a.set: 1 & {$[a \Leftarrow 1]$} \\
a.set:ai & {$[a \Leftarrow-a]$} \\
a.set:mi & {$\left[a \Leftarrow a^{-1}\right]$} \\
a.set: $\mathrm{a}\left(a^{\prime}\right)$ & {$\left[a \Leftarrow a+a^{\prime}\right]$} \\
a.set: $\mathrm{m}\left(a^{\prime}\right)$ & {$\left[a \Leftarrow a \cdot a^{\prime}\right]$} \\
a.test:0 & \\
y.cp $(a)$ & {$[y \Leftarrow a]$} \\
\hline
\end{tabular}


variable $x$ to the auxiliary variable $a, a$.set : 0 and $a$.set $: 1$ initialize the auxiliary variable $a$ to 0 and 1, respectively, $a$.set : ai and $a$.set : mi set the value of $a$ to its additive and multiplicative inverse, respectively, $a$.set : $\mathrm{a}\left(a^{\prime}\right)$ adds to the value of $a$ the value of the auxiliary variable $a^{\prime}$, a.set: $\mathrm{m}\left(a^{\prime}\right)$ multiplies the value of $a$ by the value of the auxiliary variable $a^{\prime}$, and $y \cdot \operatorname{cp}(a)$ copies the value of the auxiliary variable $a$ to the output variable $y$. Since assignment instructions always succeed, it is assumed that the returned truth value is true. An instruction of the form $a$.test : 0 is not an assignment instruction but a zero test and returns a truth value depending on the value of $a$.

\section{Examples 3.2}

1. Consider the following straight-line instruction sequence $I_{1}$ :

$$
\begin{aligned}
& a_{0} \cdot \mathrm{cp}\left(x_{0}\right) ; a_{1} \cdot \text { set }: 1 ; a_{1} . \text { set }: \mathrm{a}\left(a_{1}\right) ; a_{0} . \text { set }: \mathrm{a}\left(a_{1}\right) ; \\
& a_{1} \cdot \mathrm{cp}\left(x_{0}\right) ; a_{0} \cdot \text { set }: \mathrm{m}\left(a_{1}\right) ; a_{0} . \text { set }: \mathrm{mi} ; y \cdot \mathrm{cp}\left(a_{0}\right) ; ! \text {. }
\end{aligned}
$$

$I_{1}$ represents the total meadow mapping $x \mapsto((x+2) x)^{-1}$ : first the auxiliary variable $a_{0}$ is assigned the value of the input variable $x_{0}$ and then is raised by two, after which $a_{0}$ is multiplied by $x_{0}$, inverted and copied to the output variable $y$.

2. The periodic instruction sequence $I_{2}$

$$
\begin{aligned}
& a_{0} \cdot \mathrm{cp}\left(x_{0}\right) ; a_{1} \cdot \mathrm{cp}\left(x_{1}\right) ; a_{2} \cdot \text { set }: 1 ; a_{3} \cdot \text { set }: 1 ; a_{3} \cdot \text { set }: \mathrm{ai} ; \\
& \left(-a_{1} \text {.test }: 0 ; \# 3 ; y \cdot \mathrm{cp}\left(a_{2}\right) ; ! ; a_{2} \cdot \text { set }: \mathrm{m}\left(a_{0}\right) ; a_{1} \cdot \text { set }: \mathrm{a}\left(a_{3}\right)\right)^{\omega}
\end{aligned}
$$

represents the partial mapping $\left(x_{0}, x_{1}\right) \mapsto x_{0}^{x_{1}}$ : first, the two arguments are copied to the auxiliary variables $a_{0}$ and $a_{1}$, and $a_{2}$ and $a_{3}$ are assigned the constants 1 and -1 , respectively. In the repetition, $a_{2}$ is multiplied by the first argument, and the second argument is decreased by one until the zero test succeeds and the value of $a_{2}$ is copied to the output. This partial meadow mapping is defined for all pairs of the form $\langle x, n\rangle$.

The apply operator has been introduced in $[6,7]$ as a means to transform a given state machine or service offered by an execution environment according to a thread. Given a meadow, we view its assignments as services which can be transformed by threads as follows: a thread applied to an assignment yields - in case of terminationan assignment which assigns to the output variable the result of a computation on the input offered by the initial assignment.

Definition 3.3 Let $\mathcal{M}$ be a meadow

1. If $\alpha$ is an assignment in $\mathcal{M}$ (i.e. $\alpha \in \mathcal{M}^{\text {Var }}$ ), $v \in \operatorname{Var}$, and $m \in \mathcal{M}$, we denote by $\alpha[v:=m]$ the assignment $\alpha^{\prime}$ with

$$
\alpha^{\prime}\left(v^{\prime}\right)= \begin{cases}m & \text { if } v^{\prime} \equiv v \\ \alpha\left(v^{\prime}\right) & \text { otherwise }\end{cases}
$$


2. Let $T$ be a finite thread. We define the apply operator $T \bullet: \mathcal{M}^{\text {Var }} \cup\{\mathrm{D}\} \rightarrow \mathcal{M}^{\text {Var }} \cup$ $\{D\}$ as follows.

$$
\begin{aligned}
& T \bullet \mathrm{D}=\mathrm{D}, \\
& \mathrm{S} \bullet \alpha=\alpha, \\
& \mathrm{D} \bullet \alpha=\mathrm{D} \text {, } \\
& \left(a_{i} \cdot \operatorname{cp}\left(x_{j}\right) \circ T\right) \bullet \alpha=T \bullet \alpha\left[a_{i}:=\alpha\left(x_{j}\right)\right], \\
& \left(a_{i} \text {.set }: 0 \circ T\right) \bullet \alpha=T \bullet \alpha\left[a_{i}:=0\right] \text {, } \\
& \left(a_{i} . \text { set }: 1 \circ T\right) \bullet \alpha=T \bullet \alpha\left[a_{i}:=1\right], \\
& \left(a_{i} \text {.set: ai } \circ\right) \bullet \alpha=T \bullet \alpha\left[a_{i}:=-\alpha\left(a_{i}\right)\right] \text {, } \\
& \left(a_{i} . \text { set }: \operatorname{mi} \circ T\right) \bullet \alpha=T \bullet \alpha\left[a_{i}:=\alpha\left(a_{i}\right)^{-1}\right], \\
& \left(a_{i} \text {.set : } \mathrm{a}\left(a_{j}\right) \circ T\right) \bullet \alpha=T \bullet \alpha\left[a_{i}:=\alpha\left(a_{i}\right)+\alpha\left(a_{j}\right)\right] \text {, } \\
& \left(a_{i} \text {.set }: \mathrm{m}\left(a_{j}\right) \circ T\right) \bullet \alpha=T \bullet \alpha\left[a_{i}:=\alpha\left(a_{i}\right) \cdot \alpha\left(a_{j}\right)\right] \text {, } \\
& \left(T \unlhd a_{i} \text {.test }: 0 \unrhd T^{\prime}\right) \bullet \alpha= \begin{cases}T \bullet \alpha & \text { if } \alpha\left(a_{i}\right)=0, \\
T^{\prime} \bullet \alpha & \text { otherwise, }\end{cases} \\
& \left(y \cdot \operatorname{cp}\left(a_{j}\right) \circ T\right) \bullet \alpha=T \bullet \alpha\left[y:=\alpha\left(a_{j}\right)\right] .
\end{aligned}
$$

Threads can terminate in a number of steps depending on the input values. In the next definition we fix inductively the set $R_{T, n}^{\mathcal{M}}$ of assignments on which the thread $T$ terminates in $n$ steps. There is only a single thread that terminates in zero steps, namely $\mathrm{S}$ - and in this case, termination does not depend on the input values. In the induction step, we distinguish a couple of cases depending on the structure of the thread. Since assignment instructions always succeed, we may assume that threads corresponding to instruction sequences on rational numbers are of the form $S, D$, ins $\circ T$ or $\left(T \unlhd a_{i}\right.$.test $\left.: 0 \unrhd T^{\prime}\right)$ where ins is an assignment instruction.

Definition 3.4 Let $\mathcal{M}$ be a meadow, $T$ be a finite thread and $n \in \mathbb{N}$. $R_{T, n}^{\mathcal{M}} \subseteq \mathcal{M}^{\text {Var }}$ is defined inductively as follows.

1.

$$
R_{T, 0}^{\mathcal{M}}= \begin{cases}\mathcal{M}^{\text {Var }} & \text { if } T=\mathrm{S} \\ \varnothing & \text { otherwise }\end{cases}
$$

2.

$$
\begin{aligned}
R_{\mathrm{S}, n+1}^{\mathcal{M}} & =\mathcal{M}^{\text {Var }}, \\
R_{\mathrm{D}, n+1}^{\mathcal{M}} & =\emptyset, \\
R_{\text {insoT,n+1 }}^{\mathcal{M} \circ} & =\left\{\alpha \in \mathcal{M}^{\text {Var }} \mid(\text { ins } \circ T) \bullet \alpha \in R_{T, n}^{\mathcal{M}}\right\}, \\
R_{T \unlhd a_{i} \text {.test }: 0 \unrhd T^{\prime}, n+1}^{\mathcal{M}} & =\left\{\alpha \in R_{T, n}^{\mathcal{M}} \mid \alpha\left(a_{i}\right)=0\right\} \cup\left\{\alpha \in R_{T^{\prime}, n}^{\mathcal{M}} \mid \alpha\left(a_{i}\right) \neq 0\right\} .
\end{aligned}
$$


Lemma 3.5 Let $\mathcal{M}$ be a meadow and $T$ be a regular thread. Then for all $n \in \mathbb{N}$ and $\alpha \in R_{\pi_{n+1}(T), n}^{\mathcal{M}}$,

1. $\pi_{n+1}(T) \bullet \alpha \neq \mathrm{D}$, and

2. $\forall k>n \pi_{k}(T) \bullet \alpha=\pi_{n+1}(T) \bullet \alpha$.

Proof By straightforward induction.

We can therefore define partial mappings corresponding to regular threads as below.

Definition 3.6 Let $\mathcal{M}$ be a meadow.

1. $\alpha \in \mathcal{M}^{\text {Var }}$ is called initial if $\alpha(v)=0$ for all $v \in$ Var $-\operatorname{Var}_{\text {in }}$.

2. Let $T$ be a regular thread and $k \in \mathbb{N}$. Then $\llbracket T \rrbracket_{\mathcal{M}}^{k}: \mathcal{M}^{k+1} \stackrel{p}{\longrightarrow} \mathcal{M}$ denotes the partial mapping defined as follows:

$$
\begin{aligned}
& \mathbb{}\left[T \rrbracket_{\mathcal{M}}^{k}\left(m_{0}, \ldots, m_{k}\right)\right. \\
& \quad= \begin{cases}\left(\pi_{n+1}(T) \bullet \alpha_{m_{0}, \ldots, m_{k}}\right)(y) & \text { if } \alpha_{m_{0}, \ldots, m_{k}} \in R_{\pi_{n+1}(T), n}^{\mathcal{M}}, \\
\text { undefined } & \text { if for all } n \in \mathbb{N}, \alpha_{m_{0}, \ldots, m_{k}} \notin R_{\pi_{n+1}(T), n}^{\mathcal{M}}\end{cases}
\end{aligned}
$$

where $\alpha_{m_{0}, \ldots, m_{k}} \in \mathcal{M}^{\text {Var }}$ is the initial assignment with $\alpha\left(x_{i}\right)=m_{i}$ for $0 \leq i \leq k$ and $\alpha(v)=0$ for $v \in \operatorname{Var}-\left\{x_{0}, \ldots, x_{k}\right\}$.

Notation 3.7 If $I$ is an instruction sequence we shall write $\llbracket I \rrbracket_{\mathcal{M}}^{k}$ for the corresponding meadow mapping instead of $\llbracket|I| \rrbracket_{\mathcal{M}}^{k}$. Moreover, when dealing with partial mappings, we let the symbols $\uparrow$ and $\downarrow$ denote un- and definedness, respectively.

Example 3.8 We consider again the instruction sequences $I_{1}$ and $I_{2}$ given in Example 3.2.

1. Observe that

$$
\begin{aligned}
\left|I_{1}\right|= & a_{0} \cdot \operatorname{cp}\left(x_{0}\right) \circ a_{1} . \text { set }: 1 \circ a_{1} \cdot \text { set }: \mathrm{a}\left(a_{1}\right) \circ a_{0} \cdot \text { set }: \mathrm{a}\left(a_{1}\right) \\
& \circ a_{1} \cdot \mathrm{cp}\left(x_{0}\right) \circ a_{0} \cdot \text { set }: \mathrm{m}\left(a_{1}\right) \circ a_{0} \cdot \text { set }: \mathrm{mi} \circ y \cdot \mathrm{cp}\left(a_{0}\right) \circ \mathrm{S} .
\end{aligned}
$$

Thus

$$
\begin{aligned}
\left|I_{1}\right| \bullet \alpha= & \alpha\left[a_{0}:=\alpha\left(x_{0}\right)\right]\left[a_{1}:=1\right]\left[a_{1}:=2\right]\left[a_{0}:=\alpha\left(x_{0}\right)+2\right] \\
& {\left[a_{1}:=\alpha\left(x_{0}\right)\right]\left[a_{0}:=\left(\alpha\left(x_{0}\right)+2\right) \cdot \alpha\left(x_{0}\right)\right] } \\
& {\left[a_{0}:=\left(\left(\alpha\left(x_{0}\right)+2\right) \cdot \alpha\left(x_{0}\right)\right)^{-1}\right]\left[y:=\left(\left(\alpha\left(x_{0}\right)+2\right) \cdot \alpha\left(x_{0}\right)\right)^{-1}\right] }
\end{aligned}
$$

for every meadow $\mathcal{M}$ and every assignment $\alpha \in \mathcal{M}^{\text {Var }}$. Hence $\llbracket I_{1} \rrbracket_{\mathcal{M}}^{0}(m)=$ $((m+2) m)^{-1}$. 
2. Thread extraction of $I_{2}$ yields the regular thread $\left|I_{2}\right|$ which satisfies the equations

$$
\begin{aligned}
\left|I_{2}\right| & =a_{0} \cdot \operatorname{cp}\left(x_{0}\right) \circ a_{1} \cdot \operatorname{cp}\left(x_{1}\right) \circ a_{2} \cdot \text { set }: 1 \circ a_{3} . \text { set }: 1 \circ a_{3} . \text { set }: \text { a } \circ \circ T, \\
T & =\left(y \cdot \operatorname{cp}\left(a_{2}\right) \circ S\right) \unlhd a_{1} \text {.test }: 0 \unrhd\left(a_{2} \text {.set }: \mathrm{m}\left(a_{0}\right) \circ a_{1} \text {.set }: \mathrm{a}\left(a_{3}\right) \circ T\right) .
\end{aligned}
$$

So $\left|I_{2}\right|$ starts with five initializing actions and repeats three consecutive actions until the zero test succeeds in which case termination occurs after a final copying action. It follows that $\alpha_{m_{0}, m_{1}} \in R_{\pi_{k+1}\left(\left|I_{2}\right|\right), l}^{\mathcal{M}}$ if and only if $m_{1}=n$ for some $n \in \mathbb{N}$ and $3 n+6 \leq k, l$. Hence

$$
\llbracket I_{2} \rrbracket_{\mathcal{M}}^{1}\left(m_{0}, m_{1}\right)= \begin{cases}m_{0}^{m_{1}} & \text { if } m_{1}=n \text { for some } n \in \mathbb{N}, \\ \uparrow & \text { otherwise, }\end{cases}
$$

for every meadow $\mathcal{M}$. In case $\mathcal{M}=\mathbb{Q}, I_{2}$ yields a non-total mapping; on prime fields $\mathbb{Z} / p \mathbb{Z}$-considered zero-totalized-this mapping is total.

Every meadow mapping that is total on all meadows is clearly total on all cancellation meadows. The converse, however, does not hold: consider the instruction sequence

$$
\begin{aligned}
I= & a_{0} \cdot \operatorname{cp}\left(x_{0}\right) ;-a_{0} . \text { test }: 0 ; \# 2 ; \# 4 ; a_{0} . \text { set }: \mathrm{a}\left(a_{0}\right) ; \\
& +a_{0} . \text { test }: 0 ; \# 0 ; y \cdot \operatorname{cp}\left(a_{0}\right) ; !
\end{aligned}
$$

Given any meadow $\mathcal{M}$, we have

$$
\llbracket I \rrbracket_{\mathcal{M}}^{0}(m)= \begin{cases}0 & \text { if } m=0, \\ 0 & \text { if } m \neq 0 \& 2 m \neq 0, \\ \uparrow & \text { otherwise }\end{cases}
$$

In the absence of proper zero divisors, $m=0$ if $2 m=0$. Thus $\llbracket I \rrbracket_{\mathcal{M}}^{0}$ is the constant zero mapping on every cancellation meadow $\mathcal{M}$. On the zero-totalized field $\mathbb{Z} / 6 \mathbb{Z}$, however, $3 \neq 0$ and $2 \times 3=0$, and thus $\llbracket I \rrbracket_{\mathbb{Z} / 6 \mathbb{Z}}^{0}(3) \uparrow$.

\section{Characterization of Total Calculation on Cancellation Meadows}

In this section we shall prove the main theorem.

Meadows are standard mathematical structures, and as such, they may be described using standard logical formalisms. Here, we shall use the following first-order predicate logic over meadows and regular threads consisting of

1. the constants 0 and 1 ,

2. countably infinite constants $c_{0}, c_{1}, \ldots$,

3 . the unary function symbols - and $\stackrel{-1}{ }$, representing additive and multiplicative inversion,

4. the binary function symbols + and $\cdot$, written infix and representing addition and multiplication, 
5. for every regular thread $T$ and $k, n \in \mathbb{N}$, a $(k+1)$-ary termination predicate $R_{T, k, n}(\vec{x})$, describing the property $T$ terminates on input $x_{0}, x_{1}, \ldots, x_{k}$ after at most $n$ steps,

6. the usual Boolean connectives and first-order quantifiers with variables ranging over elements of meadows.

The standard interpretation of the termination predicates is given below.

Definition 4.1 Let $\mathcal{M}$ be a meadow. For $k, n \in \mathbb{N}$ and regular thread $T$ we define $\llbracket R_{T, k, n} \rrbracket_{\mathcal{M}} \subseteq \mathcal{M}^{k+1}$ by

$$
\llbracket R_{T, k, n} \rrbracket_{\mathcal{M}}=\left\{\left\langle\alpha\left(x_{0}\right), \ldots, \alpha\left(x_{k}\right)\right\rangle \mid \alpha \in R_{\pi_{n+1}(T), n}^{\mathcal{M}}\right\} .
$$

Example 4.2 We consider once more the instruction sequences $I_{1}$ and $I_{2}$ introduced in Examples 3.2.

1. It is easy to see that, if $I=i n s_{1} ; \ldots ; i n s_{n} ;$ ! is a straight-line instruction sequence consisting of $n$ assignment instructions and ending in a single final termination instruction, then $|I|=$ ins $_{1} \circ \cdots \circ$ ins $_{n} \circ \mathrm{S}$ and $\mathcal{M} \models \forall x_{0}, \ldots, x_{k} R_{|I|, k, n}\left(x_{0}, \ldots, x_{k}\right)$ for every meadow $\mathcal{M}$. Hence, in particular, $\mathcal{M} \models \forall x R_{\left|I_{1}\right|, 0,8}(x)$.

2. For $I_{2}$ we have for all meadows $\mathcal{M}, \mathcal{M}=\forall x R_{\left|I_{2}\right|, 1,3 n+6}(x, n)$ for all $n \in \mathbb{N}$.

Lemma 4.3 For all $k, n \in \mathbb{N}$, regular threads $T$ and meadows $\mathcal{M}$,

1. $R_{T, n}^{\mathcal{M}} \subseteq R_{T, n+1}^{\mathcal{M}}$

2. $R_{T, n}^{\mathcal{M}}=R_{\pi_{n+1}(T), n}^{\mathcal{M}}$

3. $\mathcal{M} \models \forall x_{0}, \ldots, x_{k}\left(R_{T, k, n}\left(x_{0}, \ldots, x_{k}\right) \longrightarrow R_{T, k, n+1}\left(x_{0}, \ldots, x_{k}\right)\right)$

4. $\mathcal{M} \models \forall x_{0}, \ldots, x_{k}\left(R_{T, k, n}\left(x_{0}, \ldots, x_{k}\right) \longleftrightarrow R_{\pi_{n+1}(T), k, n}\left(x_{0}, \ldots, x_{k}\right)\right)$.

Proof (1) and (2) are proven by straightforward induction; (3) and (4) follow from (1) and (2), respectively.

Recall that, in mathematical logic, the Compactness Theorem states that a set of first-order sentences has a model if and only if every finite subset has a model. This theorem is an important tool in model theory, as it provides a useful method for constructing models of any set of sentences that is finitely consistent. We use Compactness for the following finite representation property of total mappings.

Proposition 4.4 Let $T$ be a regular thread and $k \in \mathbb{N}$. If $\llbracket T \rrbracket_{\mathcal{M}}^{k}$ is total on all meadows $\mathcal{M}$, then there exists a finite thread $T^{\prime}$ such that $\llbracket T \rrbracket_{\mathcal{M}}^{k}=\llbracket T^{\prime} \rrbracket_{\mathcal{M}}^{k}$ for all meadows $\mathcal{M}$.

Proof Consider the set $\Gamma$ consisting of all meadow axioms together with the infinite set $\left\{\neg R_{T, k, n}\left(c_{0}, \ldots, c_{k}\right) \mid n \in \mathbb{N}\right\}$. If $\Gamma$ is finitely satisfiable, it must be simultaneously satisfiable, by Compactness, say in some meadow $\mathcal{M}$. This means that $\llbracket T \rrbracket_{\mathcal{M}}^{k}$ is not total, contradicting the assumption. We may therefore assume that $\Gamma$ is not finitely satisfiable. By a standard model-theoretic argument and the 
monotonicity of the termination predicate (Lemma 4.3(3)), it follows that for some $n \in \mathbb{N}$ and all meadows $\mathcal{M}, \mathcal{M} \models \forall x_{0}, \ldots, x_{k} R_{T, k, n}\left(x_{0}, \ldots, x_{k}\right)$. Hence $\mathcal{M}=$ $\forall x_{0}, \ldots, x_{k} R_{\pi_{n+1}(T), k, n}\left(x_{0}, \ldots, x_{k}\right)$ for all $\mathcal{M}$ by Lemma 4.3(4). Then $\llbracket T \rrbracket_{\mathcal{M}}^{k}=$ $\llbracket \pi_{n+1}(T) \rrbracket_{\mathcal{M}}^{k}$ for all meadows $\mathcal{M}$.

Proposition 4.5 Let $T$ be a regular thread and $k \in \mathbb{N}$. If $\llbracket T \rrbracket_{\mathcal{M}}^{k}$ is total on all cancellation meadows $\mathcal{M}$, then there exists a finite thread $T^{\prime}$ such that $\llbracket T \rrbracket_{\mathcal{M}}^{k}=\llbracket T^{\prime} \rrbracket_{\mathcal{M}}^{k}$ for all cancellation meadows $\mathcal{M}$.

Proof Repeat the previous proof with $\Gamma$ supplemented with the cancellation axiom

$$
\forall x, y, z(x \neq 0 \& x \cdot y=x \cdot z \longrightarrow y=z)
$$

We can therefore restrict ourselves to finite threads. Next we shall show that tests can be abandoned without the loss of expressive power.

For $t \in \operatorname{Ter}\left(\Sigma_{m}, \operatorname{Var}\right), \llbracket t \rrbracket_{\mathcal{M}, \alpha}$ denotes the interpretation of $t$ in the meadow $\mathcal{M}$ under the assignment $\alpha$, and if $\sigma \in \operatorname{Ter}\left(\Sigma_{m}, \operatorname{Var}\right)^{\operatorname{Var}}$, then $t^{\sigma}$ is the result of substituting all variables $v$ occurring in $t$ by $\sigma(v)$. Recall that substitutions and assignments interact in the following way.

Lemma 4.6 Let $\mathcal{M}$ be a meadow, $\alpha \in \mathcal{M}^{\text {Var }}$ an assignment and $\sigma \in \operatorname{Ter}\left(\Sigma_{m} \text {, Var }\right)^{\text {Var }}$ a substitution. Define $\alpha^{\prime} \in \mathcal{M}^{\text {Var }}$ by $\alpha^{\prime}(v)=\llbracket v^{\sigma} \rrbracket_{\mathcal{M}, \alpha}$. Then for all $t \in \operatorname{Ter}\left(\Sigma_{m}\right.$, Var $)$,

$$
\llbracket t \rrbracket_{\mathcal{M}, \alpha^{\prime}}=\llbracket t^{\sigma} \rrbracket_{\mathcal{M}, \alpha} .
$$

Proposition 4.7 Let $T$ be a finite thread and $k \in \mathbb{N}$. Then there exists a term $t_{T} \in \operatorname{Ter}\left(\Sigma_{m},\left\{x_{0}, \ldots, x_{k}\right\}\right)$ such that for all cancellation meadows $\mathcal{M}$ and all $m_{0}, \ldots, m_{k} \in \mathcal{M}$

$$
\llbracket T \rrbracket_{\mathcal{M}}^{k}\left(m_{0}, \ldots, m_{k}\right) \downarrow \longrightarrow \llbracket T \rrbracket_{\mathcal{M}}^{k}\left(m_{0}, \ldots, m_{k}\right)=\llbracket t_{T} \rrbracket_{\mathcal{M}, \alpha_{m_{0}, \ldots, m_{k}}} .
$$

Proof We use induction loading and employ structural induction on $T$ in order to prove the assertion stating the existence of a term $t_{T} \in \operatorname{Ter}\left(\Sigma_{m}\right.$, Var $)$ such that for all cancellation meadows $\mathcal{M}$ and all assignments $\alpha \in \mathcal{M}^{\text {Var }}$,

$$
(T \bullet \alpha)(y)=\llbracket t_{T} \rrbracket_{\mathcal{M}, \alpha}
$$

if $T \bullet \alpha \neq \mathrm{D}$.

If $T=\mathrm{S}$, then

$$
(\mathrm{S} \bullet \alpha)(y)=\alpha(y)=\llbracket y \rrbracket_{\mathcal{M}, \alpha} .
$$

Hence $t_{\mathrm{S}} \equiv y$. If $T=\mathrm{D}$, we also put $t_{\mathrm{D}} \equiv y$. For the induction step, we have to distinguish nine cases each of which corresponds to one of the nine instructions sorts in $\operatorname{Ins}(\mathbb{Q})$. The assignment instructions are proven straightforwardly using the previous substitution lemma. We show three cases. 
Suppose $T=a_{i} \cdot \operatorname{cp}\left(x_{j}\right) \circ T^{\prime}$ and $T \bullet \alpha \neq \mathrm{D}$. Then

$$
\begin{aligned}
(T \bullet \alpha)(y) & =\left(T^{\prime} \bullet \alpha\left[a_{i}:=\alpha\left(x_{j}\right)\right]\right)(y) \\
& =\llbracket t_{T^{\prime}} \rrbracket_{\mathcal{M}, \alpha\left[a_{i}:=\alpha\left(x_{j}\right)\right] \quad \text { by the induction hypothesis }} \\
& =\llbracket t_{T^{\prime}}^{\sigma} \rrbracket_{\mathcal{M}, \alpha} \quad \text { by Lemma } 4.6
\end{aligned}
$$

where $\sigma\left(a_{i}\right)=x_{j}$, and $\sigma(v)=v$ if $v \not \equiv a_{i}$. Hence $t_{T} \equiv t_{T^{\prime}}^{\sigma}$ suffices. Likewise, if $T=a_{i}$.set $: \mathrm{a}\left(a_{j}\right) \circ T^{\prime}$ and $T \bullet \alpha \neq \mathrm{D}$, then

$$
\begin{aligned}
(T \bullet \alpha)(y) & =\left(T^{\prime} \bullet \alpha\left[a_{i}:=\alpha\left(a_{i}\right)+\alpha\left(a_{j}\right)\right]\right)(y) \\
& =\llbracket t_{T^{\prime}} \rrbracket_{\mathcal{M}, \alpha\left[a_{i}:=\alpha\left(a_{i}\right)+\alpha\left(a_{j}\right)\right] \quad \text { by the induction hypothesis }} \\
& =\llbracket t_{T^{\prime}}^{\sigma} \rrbracket_{\mathcal{M}, \alpha} \quad \text { by Lemma } 4.6
\end{aligned}
$$

where $\sigma\left(a_{i}\right)=a_{i}+a_{j}$, and $\sigma(v)=v$ if $v \not \equiv a_{i}$. And if $T=y \cdot \operatorname{cp}\left(a_{j}\right) \circ T^{\prime}$ and $T \bullet \alpha \neq$ $D$, then

$$
\begin{aligned}
(T \bullet \alpha)(y) & =\left(T^{\prime} \bullet \alpha\left[y:=\alpha\left(a_{j}\right)\right]\right)(y) \\
& =\llbracket t_{T^{\prime}} \rrbracket_{\mathcal{M}, \alpha\left[y:=\alpha\left(a_{j}\right)\right] \quad \text { by the induction hypothesis }} \\
& =\llbracket t_{T^{\prime}}^{\sigma} \rrbracket_{\mathcal{M}, \alpha} \quad \text { by Lemma } 4.6
\end{aligned}
$$

where $\sigma(y)=a_{j}$, and $\sigma(v)=v$ if $v \not \equiv y$.

The case for the zero test exploits the fact that in every cancellation meadow $\mathcal{M}$ we have

$$
\llbracket 0_{a_{i}} \cdot t+1_{a_{i}} \cdot t^{\prime} \rrbracket_{\mathcal{M}, \alpha}= \begin{cases}\llbracket t \rrbracket_{\mathcal{M}, \alpha} & \text { if } \alpha\left(a_{i}\right)=0, \\ \llbracket t^{\prime} \rrbracket_{\mathcal{M}, \alpha} & \text { otherwise. }\end{cases}
$$

Hence, if $T=T^{\prime} \unlhd a_{i}$.test: $0 \unrhd T^{\prime \prime}$ we can take

$$
t_{T} \equiv 0_{a_{i}} \cdot t_{T^{\prime}}+1_{a_{i}} \cdot t_{T^{\prime \prime}} .
$$

The original assertion now follows from the observation that we can replace all occurrences of auxiliary variables, the output variable $y$ and all input variables $x_{n}$ with $k<n$ in the term $t_{T}$ by 0 if $\alpha$ is initial.

Definition 4.8 We shall say that the thread $T$ computes $t \in \operatorname{Ter}\left(\Sigma_{m},\left\{x_{0}, \ldots, x_{k}\right\}\right)$, if for all cancellation meadows $\mathcal{M}$ and all $m_{0}, \ldots, m_{k} \in \mathcal{M}$,

$$
\llbracket T \rrbracket_{\mathcal{M}}^{k}\left(m_{0}, \ldots, m_{k}\right)=\llbracket t \rrbracket_{\mathcal{M}, \alpha_{m_{0}, \ldots, m_{k}}} .
$$

Thus if $T$ is finite, the free variables of $t_{T}$ are among $\left\{x_{0}, \ldots, x_{k}\right\}$ and $\llbracket T \rrbracket_{\mathcal{M}}^{k}$ is total, then $T$ computes the term $t_{T}$. Conversely, every meadow term $t$ with free variables in $V_{a r}$ in can be computed by a finite thread $T_{t}$ which is in addition testfree-that is, postconditional composition occurs as action prefix only-and which uses at most five auxiliary variables. To these ends, we shall define the raise $T^{1}$ of a thread $T$ as the thread $T^{\prime}$ obtained from $T$ by raising the subscript of every auxiliary variable occurring in $T$ by one. 


\section{Definition 4.9}

1. Let $i \in \operatorname{Ins}(\mathbb{Q})$ be an instruction. Then $i^{1}$ is defined by

$$
\begin{aligned}
a_{i} \cdot \operatorname{cp}\left(x_{j}\right)^{1} & =a_{i+1} \cdot \operatorname{cp}\left(x_{j}\right), \\
a_{i} \cdot \text { set }: 0^{1} & =a_{i+1} \cdot \text { set }: 0, \\
a_{i} \cdot \text { set }: 1^{1} & =a_{i+1} \cdot \text { set }: 1, \\
a_{i} . \text { set }: \mathrm{ai}^{1} & =a_{i+1} \cdot \text { set }: \mathrm{ai}, \\
a_{i} \cdot \text { set }: \mathrm{mi}^{1} & =a_{i+1} \cdot \text { set }: \mathrm{mi}, \\
a_{i} \cdot \text { set }: \mathrm{a}\left(a_{j}\right)^{1} & =a_{i+1} \cdot \text { set }: \mathrm{a}\left(a_{j+1}\right), \\
a_{i} \cdot \text { set }: \mathrm{m}\left(a_{j}\right)^{1} & =a_{i+1} \cdot \text { set }: \mathrm{m}\left(a_{j+1}\right), \\
a_{i} \cdot \operatorname{test}: 0^{1} & =a_{i+1} \cdot \text { test }: 0, \\
y \cdot \operatorname{cp}\left(a_{j}\right)^{1} & =y \cdot \operatorname{cp}\left(a_{j+1}\right) .
\end{aligned}
$$

2. Let $T$ be a finite thread. Then $T^{1}$ is defined inductively by $\mathrm{S}^{1}=\mathrm{S}, \mathrm{D}^{1}=\mathrm{D}$, and $\left(T^{\prime} \unlhd i \unrhd T^{\prime \prime}\right)^{1}=T^{\prime 1} \unlhd i^{1} \unrhd T^{\prime \prime 1}$ for $i \in \operatorname{Ins}(\mathbb{Q})$.

A thread and its raise compute the same values.

Lemma 4.10 Let $T$ be a finite thread and $k \in \mathbb{N}$. Then for all meadows $\mathcal{M}$ and all $m_{0}, \ldots, m_{k} \in \mathcal{M}$

$$
\llbracket T \rrbracket_{\mathcal{M}}^{k}\left(m_{0}, \ldots, m_{k}\right) \downarrow \longrightarrow \llbracket T \rrbracket_{\mathcal{M}}^{k}\left(m_{0}, \ldots, m_{k}\right)=\llbracket T^{1} \rrbracket_{\mathcal{M}}^{k}\left(m_{0}, \ldots, m_{k}\right) .
$$

Proof For the sake of the proof, we define for $\alpha \in \mathcal{M}^{\text {Var }}$ the raise $\alpha^{1} \in \mathcal{M}^{\text {Var }}$ by

$$
\alpha^{1}(v)= \begin{cases}\alpha\left(a_{i+1}\right) & \text { if } v \equiv a_{i} \\ \alpha(v) & \text { if } v \notin \operatorname{Var}_{a u x}\end{cases}
$$

We now show that

$$
T \bullet \alpha^{1} \neq \mathrm{D} \longrightarrow\left(T \bullet \alpha^{1}\right)(y)=\left(T^{1} \bullet \alpha\right)(y)
$$

by structural induction on $T$. If $T=\mathrm{S}$, then

$$
\left(T \bullet \alpha^{1}\right)(y)=\left(\mathrm{S} \bullet \alpha^{1}\right)(y)=\alpha^{1}(y)=\alpha(y)=(\mathrm{S} \bullet \alpha)(y)=\left(T^{1} \bullet \alpha\right)(y) .
$$

For the induction step, we have to distinguish nine cases each of which corresponds to one of the nine instruction sorts. Each case follows straightforwardly. We show the case that $T=a_{i} \cdot \operatorname{cp}\left(x_{j}\right) \circ T^{\prime}$ :

$$
\begin{aligned}
\left(T \bullet \alpha^{1}\right)(y) & =\left(\left(a_{i} \cdot \operatorname{cp}\left(x_{j}\right) \circ T^{\prime}\right) \bullet \alpha^{1}\right)(y) \\
& =\left(T^{\prime} \bullet \alpha^{1}\left[a_{i}:=\alpha^{1}\left(x_{j}\right)\right]\right)(y)
\end{aligned}
$$




$$
\begin{aligned}
& =\left(T^{\prime} \bullet\left(\alpha\left[a_{i+1}:=\alpha\left(x_{j}\right)\right]\right)^{1}\right)(y) \\
& =\left(T^{\prime 1} \bullet \alpha\left[a_{i+1}:=\alpha\left(x_{j}\right)\right]\right)(y) \quad \text { by the induction hypothesis } \\
& =\left(a_{i+1} \cdot \operatorname{cp}\left(x_{j}\right) \circ T^{\prime 1} \bullet \alpha\right)(y) \\
& =\left(T^{1} \bullet \alpha\right)(y) .
\end{aligned}
$$

The statement now follows from the observation that $\alpha^{1}=\alpha$ if $\alpha$ is initial.

In the sequel, we shall say that a thread $T$ (an instruction sequence $I$ ) uses the auxiliary variable $a_{i}$, if the variable $a_{i}$ occurs in at least one of $T$ 's ( $I$ 's) atomic actions (basic instructions). Moreover, we shall say that $T(I)$ uses $n$ auxiliary variables, if $T(I)$ uses precisely the auxiliary variables $a_{0}, \ldots, a_{n-1}$.

\section{Lemma 4.11}

1. If $t \in \operatorname{Var}_{\text {in }} \cup\{0,1\}$, then $t$ can be computed by a finite and test-free thread that uses one auxiliary variable.

2. If t can be computed by a finite and test-free thread that uses $n$ auxiliary variables, then so can $-t$ and $t^{-1}$.

3. Suppose $t, t^{\prime} \in \operatorname{Ter}\left(\Sigma_{m}, \operatorname{Var}_{\text {in }}\right)$ can be computed by finite and test-free threads that use $n$ and $m$ auxiliary variables, respectively. If $n=m$, then $t+t^{\prime}$ and $t \cdot t^{\prime}$ can be computed by finite and test-free threads that use $n+1$ auxiliary variables, and if $n \neq m$, then $t+t^{\prime}$ and $t \cdot t^{\prime}$ can be computed by finite and test-free threads that use $\max \{n, m\}$ auxiliary variables.

Proof We shall construct appropriate threads of the form

$$
i_{1} \circ \cdots \circ i_{k} \circ y \cdot \mathrm{cp}\left(a_{0}\right) \circ \mathrm{S} .
$$

1. Observe that $a_{0} \cdot \mathrm{cp}\left(x_{i}\right) \circ y \cdot \mathrm{cp}\left(a_{0}\right) \circ \mathrm{S}, a_{0}$. set $: 0 \circ y \cdot \mathrm{cp}\left(a_{0}\right) \circ \mathrm{S}$, and $a_{0}$. set $: 1 \circ$ $y . \operatorname{cp}\left(a_{0}\right) \circ \mathrm{S}$ compute $x_{i}, 0$ and 1 , respectively.

2. Suppose $T=i_{1} \circ \cdots \circ i_{k} \circ y \cdot \mathrm{cp}\left(a_{0}\right) \circ \mathrm{S}$ computes $t$. Then

$$
i_{1} \circ \cdots \circ i_{k} \circ a_{0} . \text { set }: \text { ai } \circ y \cdot \operatorname{cp}\left(a_{0}\right) \circ \mathrm{S}
$$

computes $-t$ and

$$
i_{1} \circ \cdots \circ i_{k} \circ a_{0} . \text { set }: \mathrm{mi} \circ y \cdot \mathrm{cp}\left(a_{0}\right) \circ \mathrm{S}
$$

computes $t^{-1}$. Both threads use as many auxiliary variables as $T$ and are finite and test-free.

3. Suppose $T=i_{1} \circ \cdots \circ i_{k} \circ y \cdot \mathrm{cp}\left(a_{0}\right) \circ \mathrm{S}$ uses $n$ auxiliary variables to compute $t$, and $T^{\prime}=j_{1} \circ \cdots \circ j_{l} \circ y \cdot \mathrm{cp}\left(a_{0}\right) \circ \mathrm{S}$ uses $m$ auxiliary variables to compute $t^{\prime}$. We first assume that $n \leq m$. Since by the previous lemma $T^{1}$ also computes $t$, we have that

$j_{1} \circ \cdots \circ j_{l} \circ a_{1}$.set $: 0 \circ \cdots \circ a_{n}$. set $: 0 \circ i_{1}^{1} \circ \cdots \circ i_{k}^{1} \circ a_{0} \cdot$ set $: \mathrm{a}\left(a_{1}\right) \circ y \cdot \mathrm{cp}\left(a_{0}\right) \circ \mathrm{S}$ 
computes $t+t^{\prime}$. This thread is finite and test-free, and uses $n+1$ auxiliary variables if $n=m$ and otherwise $m$ variables. If $m<n$ then

$$
\begin{aligned}
& i_{1} \circ \cdots \circ i_{k} \circ a_{1} . \text { set }: 0 \circ \cdots \circ a_{m} \text {. set }: 0 \circ j_{1}^{1} \circ \cdots \circ j_{l}^{1} \circ a_{0} . \text { set }: \text { a }\left(a_{1}\right) \\
& \circ y \cdot \mathrm{cp}\left(a_{0}\right) \circ \mathrm{S}
\end{aligned}
$$

uses $n$ auxiliary variables and computes $t^{\prime}+t$ and hence $t+t^{\prime}$.

For $t \cdot t^{\prime}$, we replace the action $a_{0}$.set:a $\left(a_{1}\right)$ in the above threads by $a_{0}$. set $: \mathrm{m}\left(a_{1}\right)$.

Proposition 4.12 Let $t \in \operatorname{Ter}\left(\Sigma_{m}, \operatorname{Var}_{\text {in }}\right)$ be a meadow term. Then $t$ can be computed by a finite, test-free thread $T$ that uses five auxiliary variables.

Proof By Corollary 2.3, $t$ can be represented as a sum of quotients of polynomials. Moreover, every polynomial can be written as a sum of monomials, i.e. expressions of the form $n \cdot x_{i_{1}} \cdots x_{i_{k}}$ or $-n \cdot x_{i_{1}} \cdots x_{i_{k}}$. Since $n=\underbrace{1+\cdots+1}_{n}+0+0$ it can be computed by a finite and test-free thread that uses two auxiliary variables by Lemma 4.11(1) and (3). Thus also $n \cdot x_{i_{1}} \cdots x_{i_{k}}$ can all be computed by finite and testfree threads that use two auxiliary variables. And the same holds for $-n \cdot x_{i_{1}} \cdots x_{i_{k}}$ by Lemma $4.11(2)$. Thus every monomial can be computed by a finite and test-free thread that uses two auxiliary variables. It follows that every sum of monomials - and hence every polynomial — can be computed by a finite and test-free thread that uses three auxiliary variables by Lemma 4.11(3). Whence every quotient of polynomials can be computed by a finite and test-free thread that uses four auxiliary variables by Lemma 4.11(2) and (3). Invoking again Lemma 4.11(3) we obtain that every sum of quotients of polynomials - and therefore $t$ - can be computed by a finite and test-free thread that uses five auxiliary variables.

Summarizing we have proven the following completeness result.

Theorem 4.13 Let I be an instruction sequence and $k \in \mathbb{N}$ be such that $\llbracket I \rrbracket_{\mathcal{M}}^{k}$ is a total mapping on all cancellation meadows $\mathcal{M}$. Then there exists a straight-line instruction sequence $J$ which uses at most five auxiliary variables such that $\llbracket I \rrbracket_{\mathcal{M}}^{k}=$ $\llbracket J \rrbracket_{\mathcal{M}}^{k}$ for all cancellation meadows $\mathcal{M}$.

Proof Suppose that $\llbracket I \rrbracket_{\mathcal{M}}^{k}$ is total on all cancellation meadows $\mathcal{M}$. By Proposition 4.5, we can pick a finite thread $T$ such that $\llbracket I \rrbracket_{\mathcal{M}}^{k}=\llbracket T \rrbracket_{\mathcal{M}}^{k}$ for all cancellation meadows $\mathcal{M}$. By Proposition 4.7 we may assume that $T$ computes a term $t \in \operatorname{Ter}\left(\Sigma_{m},\left\{x_{0}, \ldots, x_{k}\right\}\right)$ which in turn is computed by a finite and test-free thread $T^{\prime}$ that uses five auxiliary variables by the previous proposition. We can now take a straight-line instruction sequence $J$ with $|J|=T^{\prime}$. 
Table 5 The set Signs of axioms for the sign function

$$
\begin{aligned}
& \mathbf{s}\left(1_{x}\right)=1_{x} \\
& \mathbf{s}\left(0_{x}\right)=0_{x} \\
& \mathbf{s}(-1)=-1 \\
& \mathbf{s}\left(x^{-1}\right)=\mathbf{s}(x) \\
& \mathbf{s}(x \cdot y)=\mathbf{s}(x) \cdot \mathbf{s}(y) \\
& 0_{\mathbf{s}(x)-\mathbf{s}(y)} \cdot(\mathbf{s}(x+y)-\mathbf{s}(x))=0
\end{aligned}
$$

\section{Calculation on Signed Cancellation Meadows}

We obtain signed meadows by extending the signature $\Sigma_{m}$ of meadows with the unary sign function $\mathbf{s}\left(\_\right)$. We write $\Sigma_{m s}$ for this extended signature, so $\Sigma_{m s}=$ $\left(0,1,+, \cdot,-,,^{-1}, \mathbf{s}\right)$. The sign function $\mathbf{s}$ presupposes an ordering $<$ of its domain and is defined as follows:

$$
\mathbf{s}(x)= \begin{cases}-1 & \text { if } x<0 \\ 0 & \text { if } x=0 \\ 1 & \text { if } x>0\end{cases}
$$

One can define $\mathbf{s}$ in an equational manner by the set Signs of axioms given in Table 5. First, notice that by $M d$ and axiom (1) (or axiom (2)) we find

$$
\mathbf{s}(0)=0 \quad \text { and } \quad \mathbf{s}(1)=1 .
$$

Then, observe that in combination with the inverse law $I L$, axiom (6) is an equational representation of the conditional equational axiom

$$
\mathbf{s}(x)=\mathbf{s}(y) \longrightarrow \mathbf{s}(x+y)=\mathbf{s}(x) .
$$

The initial algebra of $M d \cup$ Signs is $\mathbb{Q}$ expanded with the sign function. A proof follows immediately from the techniques used in $[10,11]$.

Some consequences of the $M d \cup$ Signs are:

$$
\begin{gathered}
\mathbf{s}\left(x^{2}\right)=1_{x} \quad \text { because } \mathbf{s}\left(x^{2}\right)=\mathbf{s}(x) \cdot \mathbf{s}(x)=\mathbf{s}(x) \cdot \mathbf{s}\left(x^{-1}\right)=\mathbf{s}\left(1_{x}\right)=1_{x}, \\
\mathbf{s}\left(x^{3}\right)=\mathbf{s}(x) \quad \text { because } \mathbf{s}\left(x^{3}\right)=\mathbf{s}(x) \cdot \mathbf{s}(x) \cdot \mathbf{s}\left(x^{-1}\right)=\mathbf{s}\left(x \cdot\left(x \cdot x^{-1}\right)\right)=\mathbf{s}(x), \\
1_{x} \cdot \mathbf{s}(x)=\mathbf{s}(x) \quad \text { because } 1_{x} \cdot \mathbf{s}(x)=\mathbf{s}\left(x^{2}\right) \cdot \mathbf{s}(x)=\mathbf{s}\left(x^{3}\right)=\mathbf{s}(x), \\
\mathbf{s}(x)^{-1}=\mathbf{s}(x) \quad \text { because } \mathbf{s}(x)^{-1}=\left(\mathbf{s}(x)^{2} \cdot \mathbf{s}(x)^{-1}\right)^{-1}=\left(\mathbf{s}\left(x^{2}\right) \cdot \mathbf{s}(x)^{-1}\right)^{-1} \\
=\left(1_{x} \cdot \mathbf{s}(x)^{-1}\right)^{-1}=1_{x} \cdot \mathbf{s}(x)=\mathbf{s}(x) .
\end{gathered}
$$

So, $0=\mathbf{s}(x)-\mathbf{s}(x)=\mathbf{s}(x)-\mathbf{s}(x)^{3}=\mathbf{s}(x)\left(1-\mathbf{s}(x)^{2}\right)$ and hence

$$
\mathbf{s}(x) \cdot(1-\mathbf{s}(x)) \cdot(1+\mathbf{s}(x))=0 .
$$


The finite basis result for the equational theory of cancellation meadows is formulated in a generic way so that it can be used for any expansion of a meadow that satisfies the propagation properties defined below.

Definition 5.1 Let $\Sigma$ be an extension of $\Sigma_{m}=\left(0,1,+, \cdot,-,{ }^{-1}\right)$, the signature of meadows. Let $E \supseteq M d$ (with $M d$ the set of axioms for meadows given in Table 3 ).

1. $(\Sigma, E)$ has the propagation property for pseudo units if for each pair of $\Sigma$-terms $t, r$ and context $C[$ ],

$$
E \vdash 1_{t} \cdot C[r]=1_{t} \cdot C\left[1_{t} \cdot r\right] .
$$

2. ( $\Sigma, E)$ has the propagation property for pseudo zeros if for each pair of $\Sigma$-terms $t, r$ and context $C[]$,

$$
E \vdash 0_{t} \cdot C[r]=0_{t} \cdot C\left[0_{t} \cdot r\right] .
$$

Preservation of these propagation properties admits the following nice result:

Theorem 5.2 (Generic Basis Theorem for Cancellation Meadows) If $\Sigma \supseteq \Sigma_{m}, E \supseteq$ $M d$ and $(\Sigma, E)$ has the pseudo unit and the pseudo zero propagation property, then $E$ is a basis (a complete axiomatization) of $\operatorname{Mod}_{\Sigma}(E \cup I L)$.

Proof See [8].

Bergstra and Ponse [8] proved that $M d$ and $M d \cup$ Signs satisfy both propagation properties and are therefore complete axiomatizations of $\operatorname{Mod}_{\Sigma}(M d \cup I L)$ and $\operatorname{Mod}_{\Sigma}(M d \cup \operatorname{Signs} \cup I L)$, respectively. Since

$$
M d \cup \text { Signs } \cup I L \vdash t=0_{t^{\prime}} \cdot s+1_{t^{\prime}} \cdot s^{\prime} \longrightarrow \mathbf{s}(t)=0_{t^{\prime}} \cdot \mathbf{s}(s)+1_{t^{\prime}} \cdot \mathbf{s}\left(s^{\prime}\right)
$$

using $I L$ and the axioms (1), (2) and (5) of Signs, it then follows that

$$
M d \cup \text { Signs } \vdash t=0_{t^{\prime}} \cdot s+1_{t^{\prime}} \cdot s^{\prime} \Longrightarrow M d \cup \operatorname{Signs} \vdash \mathbf{s}(t)=0_{t^{\prime}} \cdot \mathbf{s}(s)+1_{t^{\prime}} \cdot \mathbf{s}\left(s^{\prime}\right) .
$$

We can hence adapt the Standard Meadow Form to signed meadow terms as follows.

We write $\Sigma_{r s}=(0,1,+, \cdot,-, \mathbf{s})$ for the signature of signed rings. A signed polynomial is then an expression over $\Sigma_{r s}$, thus without inverse operator.

Definition 5.3 A term $t \in \operatorname{Ter}\left(\Sigma_{m s}, X\right)$ is a Standard Signed Meadow Form (SSMF) if, for some $n \in \mathbb{N}, t$ is an SSMF of level $n$. SSMFs of level $n$ are defined as follows:

1. SSMF of level 0: each expression of the form $s / t$ with $s$ and $t$ ranging over signed polynomials,

2. SSMF of level $n+1$ : each expression of the form

$$
0_{t^{\prime}} \cdot s+1_{t^{\prime}} \cdot t
$$

with $t^{\prime}$ ranging over signed polynomials and $s$ and $t$ over SSMFs of level $n$. 
Theorem 5.4 For each $t \in \operatorname{Ter}\left(\Sigma_{m s}, X\right)$ there exist an SSMF $t_{S S M F}$ with the same variables such that $M d \cup$ Signs $\vdash t=t_{S S M F}$.

Proof As in [8] using $(\dagger)$.

As in Corollary 2.3 it follows that every signed meadow term is provably equal to a sum of quotients of signed polynomials.

Corollary 5.5 For every $t \in \operatorname{Ter}\left(\Sigma_{m s}, X\right)$ there exist signed polynomials $s_{0}, t_{0}, \ldots$, $s_{n}, t_{n}$ such that

$$
M d \cup \text { Signs } \vdash t=\frac{s_{0}}{t_{0}}+\cdots+\frac{s_{n}}{t_{n}} .
$$

Signed polynomials also enjoy a standard form.

Lemma 5.6 Let $t$ be a signed polynomial and $n \in \mathbb{N}$ be the number of its subterms of the form $\mathbf{s}\left(t^{\prime}\right)$. Then there are polynomials $t_{1}, t_{1_{1}}, \ldots, t_{1_{n}}, \ldots, t_{i}, t_{i_{1}}, \ldots, t_{i_{n}}, \ldots, t_{3 n}$, $t_{3 n_{1}}, \ldots, t_{3 n_{n}}$ such that

$$
M d \cup \text { Signs } \vdash t=\sum_{i=1}^{3 n} \prod_{j=1}^{n} 0_{\phi\left(\mathbf{s}\left(t_{i_{j}}\right)\right)} \cdot t_{i}
$$

where $\phi\left(\mathbf{s}\left(t_{i_{j}}\right)\right) \in\left\{\mathbf{s}\left(t_{i_{j}}\right), 1+\mathbf{s}\left(t_{i_{j}}\right), 1-\mathbf{s}\left(t_{i_{j}}\right)\right\}$.

Proof We employ induction on the number $n$ of subterms of the form $\mathbf{s}\left(t^{\prime}\right)$. If $n=0$ then $t$ itself is a polynomial and hence $t_{1} \equiv t$ suffices.

Suppose $n=l+1$ and pick an innermost subterm $\mathbf{s}\left(t^{\prime}\right)$ of $t$. Then $t \equiv C\left[\mathbf{s}\left(t^{\prime}\right)\right]$ for some context $C$ and polynomial $t^{\prime}$. From $I L$ together with (11) it follows that $\mathbf{s}\left(t^{\prime}\right)=0$ or $\mathbf{s}\left(t^{\prime}\right)=1$ or $\mathbf{s}\left(t^{\prime}\right)=-1$. Thus

$$
M d \cup \text { Signs } \vdash t=0_{\mathbf{s}\left(t^{\prime}\right)} \cdot C[0]+0_{1-\mathbf{s}\left(t^{\prime}\right)} \cdot C[1]+0_{1+\mathbf{s}\left(t^{\prime}\right)} \cdot C[-1]
$$

with $C[0], C[1]$, and $C[-1]$ having $l$ signed subterms. We can now apply the induction hypothesis.

A suitable instruction for computations on signed meadows is $a$.set:s with Boolean reply true and the obvious semantics $a \Leftarrow \mathbf{s}(a)$. We add this instruction to $\operatorname{Ins}(\mathbb{Q})$, and consider instruction sequences and corresponding threads over the enriched instruction set in the sequel.

Example 5.7 Notice that, with the sign function available, the function $\max \left(x_{0}, x_{1}\right)$ has the following simple definition

$$
\max \left(x_{0}, x_{1}\right)= \begin{cases}\left(\mathbf{s}\left(x_{0}\right)+1\right) \cdot x_{0} / 2 & \text { if } x_{1}=0 \\ \max \left(x_{0}-x_{1}, 0\right)+x_{1} & \text { otherwise }\end{cases}
$$


The function $\max (x, y)$ can be computed by the periodic instruction sequence

$$
\begin{aligned}
& a_{0} . \mathrm{cp}\left(x_{0}\right) ; a_{1} \cdot \mathrm{cp}\left(x_{1}\right) ; a_{2} . \text { set }: 0 ; a_{4} \cdot \mathrm{cp}\left(x_{0}\right) ; a_{1} \text {.test }: 0 ; \# 2 ; \# 11 ; \\
& \left(a_{3} . \text { set }: 1 ; a_{4} . \text { set }: \mathrm{s} ; a_{4} . \text { set }: \mathrm{a}\left(a_{3}\right) ; a_{0} . \text { set }: \mathrm{m}\left(a_{4}\right) ;\right. \\
& a_{3} . \text { set }: \mathrm{a}\left(a_{3}\right) ; a_{3} . \text { set }: \mathrm{mi} ; a_{0} . \text { set }: \mathrm{m}\left(a_{3}\right) ; a_{0} . \text { set }: \mathrm{a}\left(a_{2}\right) ; y \cdot \mathrm{cp}\left(a_{0}\right) ; \text { !; } \\
& \left.a_{1} . \text { set }: \mathrm{ai} ; a_{0} . \text { set }: \mathrm{a}\left(a_{1}\right) ; a_{4} . \text { set }: \mathrm{a}\left(a_{1}\right) ; a_{2} \cdot \mathrm{cp}\left(x_{1}\right)\right)^{\omega}
\end{aligned}
$$

which also has a finite representation.

The termination predicate and the apply operator can both be extended to regular threads using the sign instruction in the obvious way by defining

$$
\begin{aligned}
R_{a_{i} . \text { set }: \mathrm{s} \circ T, n+1}^{\mathcal{M}} & =\left\{\alpha \in \mathcal{M}^{\text {Var }} \mid \alpha\left[a_{i}:=\mathbf{s}\left(\alpha\left(a_{i}\right)\right)\right] \in R_{T, n}^{\mathcal{M}}\right\}, \quad \text { and } \\
\left(a_{i} . \text { set }: \mathrm{s} \circ T\right) \bullet \alpha & =T \bullet \alpha\left[a_{i}:=\mathbf{s}\left(\alpha\left(a_{i}\right)\right)\right] .
\end{aligned}
$$

We then have the following completeness result.

Theorem 5.8 Let I be an instruction sequence and $k \in \mathbb{N}$ be such that $\llbracket I \rrbracket_{\mathcal{M}}^{k}$ is a total mapping on all signed cancellation meadows $\mathcal{M}$. Then there exists a straightline instruction sequence $J$ which uses at most eight auxiliary variables such that $\llbracket I \rrbracket_{\mathcal{M}}^{k}=\llbracket J \rrbracket_{\mathcal{M}}^{k}$ for all cancellation meadows $\mathcal{M}$.

Proof The Propositions 4.5 and 4.7 extend straightforwardly to signed cancellation meadows. Thus $|I|$ computes a term $t \in \operatorname{Ter}\left(\Sigma_{m s},\left\{x_{0}, \ldots, x_{k}\right\}\right)$. It remains to show that $t$ can be computed by a finite and test-free thread that uses at most eight auxiliary variables. From Corollary 5.5 it follows that $t$ is provably equal to a sum of quotients of signed polynomials. Then, following the proof of Proposition 4.12, it suffices to prove that a signed polynomial can be computed by a finite and test-free thread using at most six auxiliary variables. To these ends, we invoke Lemma 5.6. Thus we may assume that there exist polynomials $t_{1}, t_{1_{1}}, \ldots, t_{1_{n}}, \ldots, t_{i}, t_{i_{1}}, \ldots, t_{i_{n}}, \ldots, t_{3 n}, t_{3 n_{1}}, \ldots, t_{3 n_{n}}$ such that

$$
t=\sum_{i=1}^{3 n} \prod_{j=1}^{n} 0_{\phi\left(\mathbf{s}\left(t_{i j}\right)\right)} \cdot t_{i}
$$

where $\phi\left(\mathbf{s}\left(t_{i_{j}}\right)\right) \in\left\{\mathbf{s}\left(t_{i_{j}}\right), 1+\mathbf{s}\left(t_{i_{j}}\right), 1-\mathbf{s}\left(t_{i_{j}}\right)\right\}$.

From Lemma 4.11 it follows that a polynomial $t^{\prime}$ can be computed by a finite and test-free thread using three auxiliary variables. Say

$$
i_{1} \circ \cdots \circ i_{k} \circ y \cdot \mathrm{cp}\left(a_{0}\right) \circ \mathrm{S}
$$

computes $t^{\prime}$. Then

$$
i_{1} \circ \cdots \circ i_{k} \circ a_{0} . \text { set: } \mathrm{s}\left(a_{0}\right) \circ y \cdot \mathrm{cp}\left(a_{0}\right) \circ \mathrm{S}
$$

computes $\mathbf{s}\left(t^{\prime}\right)$ using the same variables. Thus also $\phi\left(\mathbf{s}\left(t^{\prime}\right)\right)$ can be computed by a finite and test-free thread using three auxiliary variables. Hence $0_{\phi\left(\mathbf{s}\left(t_{i_{j}}\right)\right)} \cdot t_{i}$ can be 
computed with four auxiliary variables by a finite and test-free thread. Therefore it takes at most five auxiliary variables to compute $\Pi_{j=1}^{n} 0_{\phi\left(\mathbf{s}\left(t_{i}\right)\right)} \cdot t_{i}$ and six to compute $t$ by a finite thread without any tests.

\section{Conclusions and Future Work}

We have described an algebraic execution system that can be used to analyze properties of instruction sequences. It is especially designed to perform calculation on the signed rational numbers. We have proven that total instruction sequences can be computed by straight-line programs with a bound supply of auxiliary variables.

By itself the standard forms that we have derived have no practical merit. But they indicate that tests, jumps and loops are primarily needed for reasons of complexity reduction. We have no proofs available that by using tests or jumps or larger numbers of auxiliary variables the running time of programs that compute total functions over cancellation meadows can be shortened, but that question clearly merits further attention. Using instruction sequences with tests, forward jumps and backward jumps to represent a given computable function many forms of optimization can be sought. Code compactness is optimized by having a relatively low number of instructions, run time complexity is optimized if programs terminate after relatively few steps. Several other aspects can be investigated at this level of abstraction: the degree to which a program profits from pipe-lined execution, and the optimal pipe-line design for its execution. More generally the degree to which a program admits a multi-threaded execution can be studied.

A second issue concerns the expandability of the data type. Important computer algorithms based on discrete Fourier transformations can be expressed within the signed rational numbers extended with sin and $\pi$. For future work, we aim at examining equivalence and simplification problems for this kind of straight-line instruction sequences. However, it is yet unclear to us where straightening starts to fail.

Open Access This article is distributed under the terms of the Creative Commons Attribution Noncommercial License which permits any noncommercial use, distribution, and reproduction in any medium, provided the original author(s) and source are credited.

\section{References}

1. Bergstra, J.A., Bethke, I.: Polarized process algebra and program equivalence. In: Baeten, J.C.M., Lenstra, J.K., Parrow, J., Woeginger, G.J. (eds.) Automata, Languages and Programming, 30th International Colloquium, ICALP 2003, Eindhoven, The Netherlands, June 30-July 4. LNCS, vol. 2719, pp. 1-21. Springer, Berlin (2003)

2. Bergstra, J.A., Bethke, I., Ponse, A.: Decision problems for pushdown threads. Acta Inform. 44(2), 75-90 (2007)

3. Bergstra, J.A., Hirschfeld, Y., Tucker, J.V.: Meadows and the equational specification of division. Theor. Comput. Sci. 410(12-13), 1261-1271 (2009)

4. Bergstra, J.A., Loots, M.E.: Program algebra for sequential code. J. Logic Algebr. Program. 51(2), 125-156 (2002)

5. Bergstra, J.A., Middelburg, C.A.: Thread algebra for strategic interleaving. Form. Asp. Comput. 19(4), 445-474 (2007) 
6. Bergstra, J.A., Middelburg, C.A.: Instruction sequence processing operators (2009). arXiv:0909.2088

7. Bergstra, J.A., Ponse, A.: Combining programs and state machines. J. Logic Algebr. Program. 51, 175-192 (2002)

8. Bergstra, J.A., Ponse, A.: A generic basis theorem for cancellation meadows (2008). arXiv:0803.3969v2

9. Bergstra, J.A., Ponse, A., van der Zwaag, M.B.: Tuplix calculus. Sci. Ann. Comput. Sci. 18, 35-61 (2008)

10. Bergstra, J.A., Tucker, J.V.: The rational numbers as an abstract data type. J. ACM 54(2) (2007)

11. Bethke, I., Rodenburg, P.: The initial meadows. J. Symb. Log., September, 2010

12. Bethke, I., Rodenburg, P., Sevenster, A.: The structure of finite meadows (2009). arXiv:0903.1196

13. Bui, D.B., Mavlyanov, A.V.: Theory of program algebras. Ukr. Math. J. 36(6), 761-764 (1984)

14. Bui, D.B., Mavlyanov, A.V.: Mutual derivability of operations in program algebra. I. Cybern. Syst. Anal. 24(1), 35-39 (1988)

15. Bui, D.B., Mavlyanov, A.V.: Mutual derivability of operations in program algebra. II. Cybern. Syst. Anal. 24(6), 1-6 (1988)

16. Ibarra, O.A., Moran, S.: Probabilistic algorithms for deciding equivalence of straight-line programs. J. Assoc. Comput. Mach. 30(1), 217-228 (1983)

17. Ibarra, O.A., Leininger, B.S.: On the simplification and equivalence problems for straight-line programs. J. Assoc. Comput. Mach. 30(3), 641-656 (1983)

18. Ponse, A., van der Zwaag, M.B.: An introduction to program and thread algebra. In: Beckmann, A., et al. (eds.) Logical Approaches to Computational Barriers: Proceedings CiE 2006. LNCS, vol. 3988, pp. 445-458. Springer, Berlin (2006)

19. von Wright, J.: An interactive metatool for exploring program algebras. Turku Centre for Computer Science, TUCS Technical Report No. 247, March, 1999 